\title{
COMUNIDAD MEXICANA DE HISTORIADORES DE LA EDUCACIÓN. APROXIMACIONES A UN RECUENTO HISTORIOGRÁFICO 2002-2012
}

\author{
Mexican Community of Historians of Education.
} Towards an historiographical account of the years 2002-2012

\author{
María Esther Aguirre Lora ${ }^{\S}$ y Jesús Márquez Carrillo* \\ Fecha de recepción: 15/05/2016 • Fecha de aceptación: 21/09/2016
}

Resumen. El Consejo Mexicano de Investigación Educativa (COMIE) organiza cada diez años la realización de estados del conocimiento en diecisiete áreas temáticas. La actividad se dirige a realizar un estado de la cuestión con el fin de descubrir las tendencias de la década y analizarlas. Como participantes directos en el último estado de conocimiento relativo a la historia y la historiografía de la educación, este artículo se propone -en líneas generales- dar cuenta del proceso seguido para recabar la información, así como mostrar en gruesos trazos las tendencias globales percibidas en la última década (2002-2012). Por su carácter general no entra en detalles, pero tiene el respaldo de la vasta producción historiográfica que se detalla en el cuerpo del artículo.

Palabras clave: México; Comunidad historiadores de la educación; Historiografía de la educación; Producción académica; Estado del conocimiento.

Abstract. Every ten years the Mexican Council of Education Research organizes the gathering of information for the "state of knowledge» in seventeen different areas of study. The aim of this activity is to assess the "state of knowledge» in order to discover and to analyze the main tendencies of the decade.

Having been directly involved in the gathering of the last «state of knowledge» in relation to the sub-area of History and Historiography of Education, the purpose of this article is to give an account, in general lines, of the processes we followed to collect the information, as well as the global tendencies in the last decade (2002-2012). Given its general nature, the article does not

\footnotetext{
$\S$ Instituto de Investigaciones sobre la Universidad y la Educación. Universidad Nacional Autónoma de México. Lado norte de la Sala de Conciertos Nezahualcóyotl. Centro Cultural Universitario. Delegación Coyoacán. 04510 México, D. F. México. mariaestheraguirre@gmail.com

"Facultad de Filosofía y Letras. Benemérita Universidad Autónoma de Puebla. Juan de Palafox y Mendoza 227-229. Centro Histórico. 72000 Puebla. México. jesusm146@hotmal.com
}

Cómo citar este artículo: Aguirre Lora, M. Esther y Márquez Carrillo, Jesús. «Comunidad mexicana de historiadores de la educación. Aproximaciones a un recuento historiográfico 2002-2012», Historia y Memoria de la Educación, 5 (2017): 401-422. 
focus on so much on particularities, but rather deals with the central historiographic production of recent years.

Key words: Mexico; Community of historians of education; Historiography of education; Academic production; State of knowledge.

La investigación educativa en el mundo occidental como concepto y como campo de estudio no procede de años recientes. Sus orígenes se remontan al último tramo del siglo XIX, y surgió de la pedagogía y de la psicología con una orientación práctica, enfocada al estudio de los factores que incidían en el quehacer escolar en sus diferentes aspectos y en sus distintos actores. Fue experimental, sustentada en el paradigma positivista.

En el caso de México, desde los años veinte podemos rastrear experiencias que resultaron muy reconocidas y que se pueden profundizar en otro contexto. Interesa destacar al respecto que estas iniciativas fueron el paso obligado para configurar el interés por la investigación educativa.

En los años sesenta y setenta, con el proceso de expansión de la matrícula - y una importante diversificación de la oferta educativa- surgen instancias abocadas a la investigación educativa. Luego, en la década de los años ochenta, el incremento de organizaciones e instituciones orientadas a la investigación educativa contribuyeron en gran modo al crecimiento de comunidades constituidas alrededor de la misma. La creación del Programa Nacional Indicativo de Investigación Educativa del Consejo Nacional de Ciencia y Tecnología (1980) y del Plan Maestro de Investigación Educativa, inscrito en él (1982-1984), el establecimiento del Sistema Nacional de Investigadores (1984), la fundación del Consejo Mexicano de Investigación Educativa (1993), los Congresos Nacionales de Investigación Educativa, ${ }^{1}$ el apoyo al desarrollo de los posgrados, entre otros, son algunos elementos que resultaron favorables para potenciar esta actividad, incluso con orientaciones distintas. De ahí surgen los estados del conocimiento acerca de lo producido en un campo específico de los estudios en educación. En este contexto, el Consejo Mexicano de Investigación Educativa (COMIE) ha promovido cada diez años la realiza-

\footnotetext{
${ }^{1}$ Eduardo Weiss, El campo de la investigación educativa en México a través de los estados de conocimiento, Conferencia magistral, VIII Congreso Nacional de Investigación Educativa (2005); Alicia Colina Escalante, «El crecimiento del campo de la investigación educativa en México. Un análisis a través de sus agentes», Perfiles Educativos, XXXIII (132), (2011): 10-28.
} 
ción de estados del conocimiento organizados en diecisiete subcampos o áreas temáticas. ${ }^{2}$

\section{VICISITUDES Y RESULTADOS}

En el curso del trabajo realizado la categoría que empleamos para ir vertebrando los avances fue la de campo, tanto en la perspectiva bourdiana como en un sentido próximo a la noción anglosajona. En el primer caso la noción es entendida como campo de fuerzas y toma de posición en torno a un capital cultural, donde está presente el conflicto; ${ }^{3}$ en el segundo, el concepto se concibe como un «campo de estudios», quitándole gravidez al espacio de lucha en torno a un capital cultural para dar cuenta de un lugar donde convergen distintas tradiciones histórico-culturales en constante movimiento y amalgama unas con otras. Así mismo hemos asumido la noción de tribus y territorios, ${ }^{4}$ que a su vez nos remite al problema de las configuraciones e identidades gestadas en torno a la producción de conocimiento. De hecho, podemos decir que en la actualidad los historiadores de la educación están más próximos a una idea de un grupo de estudiosos, con distintas inserciones institucionales y de dedicación al campo de la historia de la educación, pero que, finalmente, convergen en la necesidad de someter a debate las prácticas referidas a la indagación y a la escritura de la historia, donde se ponen en juego los hilos sutiles entre historia e historiografía de la educación.

Una característica particular de nuestro trabajo en relación con los realizados en años anteriores $(1992,2002)$ fue la propuesta de realizar

\footnotetext{
${ }^{2}$ Nos referimos a: 1. Aprendizaje y desarrollo humanos; 2. Currículo; 3. Educación ambiental para la sustentabilidad; 4. Educación superior, ciencia y tecnología; 5 . Educación y conocimientos disciplinares; 6. Educación y valores; 7. Entornos virtuales de aprendizaje; 8. Filosofía, Teoría y Campo de la educación; 9. Historia e historiografía de la educación; 10. Relaciones entre educación y mercados laborales; 11 . Otras relaciones entre educación y sociedad; 12 . Investigación de la investigación educativa; 13. Multiculturalismo y educación; 14. Política y gestión; 15. Prácticas educativas en espacios escolares; 16. Procesos de formación; 17. Sujetos de la educación; 18. Convivencia, disciplina $\mathrm{y}$ violencia en las escuelas.

${ }^{3}$ Martiniano Arredondo y otros, «La investigación educativa en México. Un campo científico en proceso de constitución», Revista Mexicana de Sociología, XLVI (1), (1984): 5-38; Martiniano Arredondo y otros, Los procesos de formación y conformación de los agentes de la investigación educativa (México: UNAM-CESU, 1989).

${ }^{4}$ Tony Becher, Tribus y territorios académicos: la indagación intelectual y las culturas de las disciplinas (Barcelona: Gedisa, 2001).
} 
una base de datos susceptible de consultarse desde cualquier lugar y por cualquier persona interesada en ella. Los productos por reunir fueron libros, capítulos de libro, artículos de revistas, revistas, ponencias, memorias, publicaciones electrónicas y tesis de posgrado.

Al concluir el trabajo, logramos la participación directa de 18 estados de la República y de un sector muy pequeño de la zona metropolitana de la capital del país, lo cual representa un poco más de la mitad de todo el país: $59,37 \%$, porcentaje que no refleja la totalidad de los estados cuya producción fue posible detectar, puesto que para varias entidades se logró un acercamiento a su producción a través de otras vías, como el rastreo en bases de datos y diversos repositorios. Esta situación elevó el porcentaje de información registrada, procedente de distintos estados, al 84,37\%.

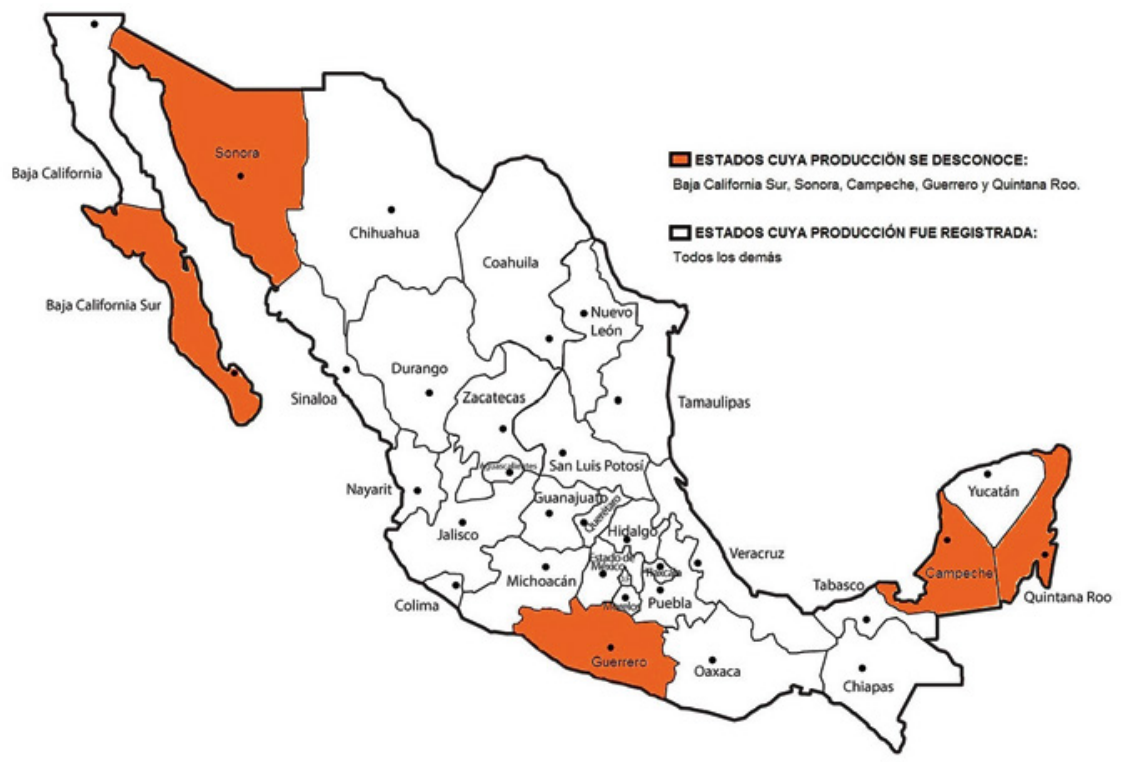

Somos conscientes de que los registros realizados en la base de datos son incompletos, y, en todo caso, son susceptibles de mejora. La tarea ha sido muy compleja y lo que presentamos es el resultado del trabajo de un conjunto de colegas que creímos y coincidimos en la viabilidad e importancia del proyecto pese a las adversidades.

En este sentido, la gran aportación de este enorme esfuerzo es el haber iniciado una base de datos sobre nuestro trabajo, abierta, susceptible de 
consultarse y enriquecerse en años sucesivos. Es producto, fundamentalmente, de nuestros historiadores de la educación procedentes de las distintas regiones del país y del grupo de trabajo que logramos constituir, el cual, sumando todos ellos, da un resultado de 85 personas con tareas específicas.

Una vez recabada y registrada la información sobre la producción, con el propósito de apoyar la siguiente fase de trabajo que consistía en el análisis, elaboramos, a manera de sugerencia, un listado de indicadores para cada uno de los productos. Aunque hubo criterios básicos, cada grupo, desde su propia óptica y forma de trabajo, dio diferentes soluciones: unos organizaron el análisis por región; otros, por tema. No hubo categorías de análisis fijas; sólo criterios muy generales con algunas palabras-clave que pudieran orientar los registros y su ulterior interpretación. Estas fueron:

- Enfoques.

- Instituciones (formales y no formales).

- Comunidades sociales, comunidades académicas, comunidades culturales.

- Cultura escolar.

- Agente, sujeto, actor.

- Enseñanza de la historia.

- Archivos y fuentes.

Pensamos que todo esto, finalmente, expresa diversas formas de hacer historia de la educación.

La tarea resultó titánica, como puede constatarse en el siguiente cuadro y su correspondiente gráfica:

\begin{tabular}{|l|c|}
\hline \multicolumn{1}{|c|}{ Productos } & $\begin{array}{c}\text { Totales } \\
\text { registrados }\end{array}$ \\
\hline Libros y capítulos & 768 \\
\hline Artículos en revistas & 367 \\
\hline Ponencias en memorias & 1203 \\
\hline Memorias de congresos & 131 \\
\hline Tesis & 244 \\
\hline Publicaciones electrónicas & 31 \\
\hline Totalidad de registros & 2744 \\
\hline
\end{tabular}




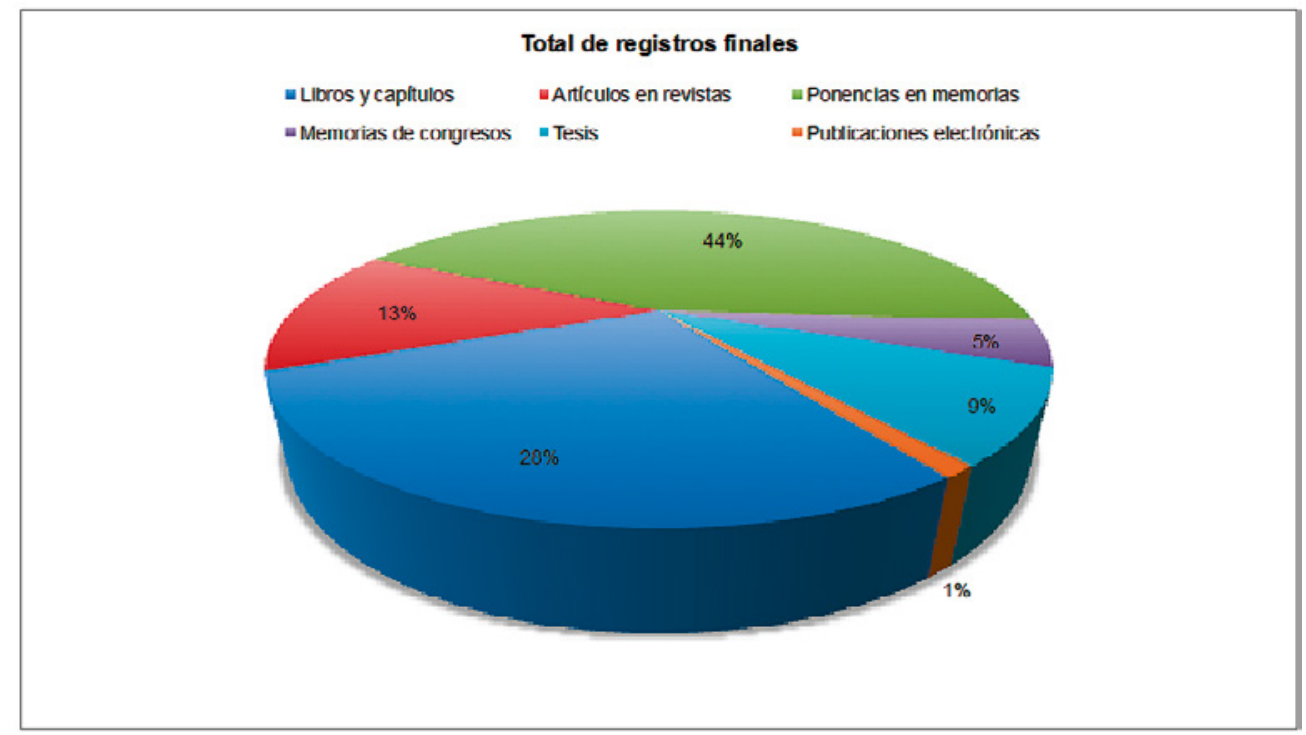

\section{DESPLIEGUE DEL CAMPO DISCIPLINAR}

Desde el último tercio del siglo XIX, la escritura de la historia de la educación quedó en manos de eruditos, políticos, pedagogos y pensadores en general, poco versados en métodos y técnicas de investigación, diletantes, testigos de una época y constructores del proyecto de país, como hombres de acción que incidían en la vida política en cualesquiera de sus esferas o bien vinculados con el ámbito escolar.

Pero los años setenta del pasado siglo marcaron un parteaguas en las formas de producción en este campo. Hasta principios de esos años, los historiadores de la educación —si se dedicaban a la época colonial y eran profesionales - seguían el modelo trazado por Joaquín García Icazbalceta (1825-1894), en la perspectiva de un empirismo erudito; interpretaban los procesos educativos del país influidos por la antropología norteamericana y los lineamientos más generales del marxismo o, adscritos a la filosofía historicista, se dedicaban a explorar la historia de las ideas y la vida de las instituciones escolares. ${ }^{5}$ En este último aspecto es de subra-

\footnotetext{
${ }^{5}$ Enrique González González, «Historia de la educación en la época colonial», Historia Mexicana, XLIII (2), (1993): 345-351; Álvaro Matute, La teoría de la historia en México (1940-1973) (México: Secretaría de Educación Pública, Colección Sep/setentas núm. 126, 1974); Guillermo de la Peña, «Instituciones
} 
yar la presencia en El Colegio de México de José Gaos (1900-1969), José Miranda (1903-1967) y Silvio Zavala (1909-2014), quienes fundaron un seminario al respecto en los años cuarenta y cincuenta. Este seminario, bajo la dirección de José Gaos, rindió excelentes frutos; el magisterio de Miranda y Zavala estimuló el análisis de las instituciones. ${ }^{6}$ De ahí surgió un conjunto de estudios sobre el nacimiento y desarrollo de la filosofía moderna en el mundo de habla hispana, que apuntaban hacia la historia intelectual y la historia de las ideas, y también trabajos monográficos memorables. Entre ellos destacan trabajos sobre la organización de los estudios en la Nueva España (Becerra López, 1963), los métodos pedagógicos de los jesuitas (Xavier Gómez Robledo, 1954) y sus intereses económicos en la actividad evangelizadora (Raúl Flores Guerrero, 1954), la historia del Colegio de Santa Cruz Tlatelolco (José María Kobayashi, 1974) y el Seminario de Valladolid a fines del siglo XVIII (Agustín García Alcaraz, 1971), o la historia de la educación en Guadalajara durante la época colonial (Carmen Castañeda, 1974), por citar sólo algunas obras. ${ }^{7}$

Sin embargo, fue en el seno de las ciencias sociales y políticas donde comenzó a emerger, a principios de los años setenta, la historiografía crítica de la educación. Esta forma de hacer historia planteó que la escuela y el aparato escolar no eran sólo un instrumento de desarrollo social, sino también un dispositivo de reproducción, control y conflicto. ${ }^{8}$ Situada en el campo de la lucha de clases, su propósito fue coadyuvar en la formación y el desarrollo de una sociedad democrática. Y en esta búsqueda congregó a historiadores, sociólogos y antropólogos, quienes desde 1973 formaron el Seminario de Educación en el CIS-INAH (Centro de Investigaciones Superiores del Instituto Nacional de Antropología e Historia), hoy CIESAS-Mé-

educativas», en Balance y perspectivas de la historiografía social en México (México: Instituto Nacional de Antropología e Historia, 1979), 199-227, 215-217 y 219); Clara Inés Ramírez González, «La Real Universidad de México en los siglos XVI y XVII. Enfoques recientes», en Historia de las universidades modernas en Hispanoamérica. Métodos y fuentes (México: UNAM, 1995), 275-280.

${ }^{6}$ El seminario se llamó Seminario para el Estudio del Pensamiento en los Países de Lengua Española [Matute, La teoría de la historia en México (1940-1973), 16-17].

${ }^{7}$ De la Peña, «Instituciones educativas», 215 y 217.

${ }^{8}$ La elaboración de estas ideas fue deudora de los movimientos de liberación nacional, un marxismo renovador (Louis Althusser, Nicos Poulantzas, Antonio Gramsci, José Carlos Mariátegui) y las teorías de la dependencia (André Gunder Frank, Fernando Henrique Cardoso, Enzo Faletto). Vid. Susana Quintanilla Osorio, «Panorama actual de la historia de la educación en México», en Historia de la educación: perspectivas de análisis (México: Instituto Superior de Ciencias de la Educación del Estado de México, 1991), 9-18 (12-14). 
xico (Centro de Investigaciones y Estudios Superiores en Antropología Social-México), donde pretendieron dar soporte intelectual a ese proyecto. Luego, sus ideas se fueron transformando con el surgimiento de la profesión académica, ${ }^{9}$ el cambio del contexto político nacional, la especialización disciplinar y la investigación cualitativa sobre las escuelas.

En la década de los ochenta, varios investigadores -principalmente del DIE-CINVESTAV (Departamento de Investigaciones Educativas del Centro de Investigaciones y Estudios Avanzados) - se propusieron esclarecer en la investigación educativa sobre las escuelas los procesos de reproducción social, resistencia y apropiación culturales a una escala menor, más densa y/o profunda. ${ }^{10}$ En su perspectiva, la experiencia escolar está atravesada por múltiples y complejos procesos que son la base para la construcción social de la escuela y del conocimiento. A diferencia de la historiografía crítica de la educación, este enfoque se reveló de capital importancia para interpretar y comprender las transformaciones que individual y socialmente se generan en el mundo cotidiano de la escuela y el aula, en la cultura escolar. Por eso, hoy su estudio resulta imprescindible si queremos comprender históricamente las distintas dimensiones y espacios del quehacer educativo.

Un poco antes, a principios de los años setenta, surgió en El Colegio de México el Seminario de Historia de la Educación en México, que desde 1976 se convirtió en un taller. ${ }^{11}$ Ahí, Dorothy Tanck propuso hacer una historia social de la educación que diera cuenta de los procesos internos en las escuelas, la procedencia geográfica de los estudiantes, el porqué de los cambios en cada uno de los distintos niveles educativos, las relaciones entre educación e ideas políticas, sistema escolar y sistema político,

\footnotetext{
${ }^{9}$ Nos referimos a un concepto procedente de la tradición anglosajona que coloca en el centro del debate a las profesiones universitarias como matriz formadora de la que surgen las demás profesiones. Remite al proceso de reconfiguración de la profesión que, en décadas relativamente recientes, plantea nuevas formas de regulación social, de certificación de habilidades y dominio del campo, de condiciones de producción intelectual, lo cual se expresa en importantes cambios identitarios.

${ }^{10}$ En cuanto a sus orientaciones teóricas y metodológicas, fue evidente en sus inicios la lectura de Agnes Heller; luego vendría la antropología interpretativa y el interaccionismo simbólico, además de las teorías de la reproducción y de la resistencia.

${ }^{11}$ Luz Elena Galván Lafarga y Susana Quintanilla Osorio, Historiografía de la Educación (México: Consejo Mexicano de Investigación Educativa, 1993), 9-11. Sobre los temas, fuentes y formas de trabajar en dicho Seminario, véase Valentina Torres Septién, «La experiencia del seminario de historia de la educación en México de El Colegio de México», en Historia de la Educación: perspectivas de análisis, 19-29.
} 
etcétera. Con todo, fiel a sus orígenes, este Seminario se inclinó más por estudiar a la educación en sus contextos. ${ }^{12}$ Pilar Gonzalbo -entre otras investigadoras - ha subrayado la importancia de investigar los nexos de la educación con la historia de la vida cotidiana, la familia, la vida privada, la mujer, la sexualidad y, en suma, la historia de las mentalidades. ${ }^{13}$

Hoy es impensable la historia de la educación en México sin la experiencia de ese Seminario, cuyo enraizamiento académico se ha extendido hacia varias ciudades del país (Toluca, Guadalajara, Puebla, Zacatecas), principalmente a través de sus graduados. Sus contribuciones han servido para interpretar y comprender distintos aspectos y procesos de la educación mexicana.

Por otra parte, desde su apertura en 1976, el Centro de Estudios sobre la Universidad de la UNAM impulsó la realización de investigaciones sobre los estudios superiores. ${ }^{14}$ El Seminario de Historia Colonial comenzó a indagar la vida de la Universidad a partir de sus propias especificidades. Orientado en sus inicios hacia el estudio de la universidad colonial, el Seminario amplió sus perspectivas temáticas ante

la necesidad de comprender el papel de la corporación universitaria dentro del marco general de la educación y la sociedad novohispanas, y pronto incluyó dentro de sus estudios a los colegios y seminarios e, incluso, se vio precisado a extender sus miras cronológicas, a fin de captar la transición del fenómeno educativo más allá de la época colonial, llegando hasta las reformas del siglo XIX. ${ }^{15}$

\footnotetext{
${ }^{12}$ La historia de la educación debe ser vista «como la columna vertebral de la historia que necesita ser analizada dentro del contexto de la historia intelectual, política y social del momento que se analiza», dice Torres Septién (Valentina Torres Septién, «Comentario», en Memorias del Simposio de historiografía mexicanista (México: CMCH, Gobierno del Estado de Morelos, IIH-UNAM, 1990), 699-705 (700).

${ }^{13}$ Pilar Gonzalbo, «La vida cotidiana en el México colonial», en Ideas, valores y tradiciones. Ensayos sobre historia de la educación en México (México: El Colegio Mexiquense, 1996), 67-77 (69). Para abordar estos temas y problemas de investigación, en 1979 surgió, en el Departamento de Estudios Históricos del INAH, surgió el Seminario de Historia de las Mentalidades y Religión en el México Colonial. Sobre los orígenes, alcances y limitaciones de este seminario, véase Pilar Gonzalbo, «Los límites de las mentalidades», en Memorias del Simposio de Historiografía Mexicanista, 475-486.

${ }^{14}$ Cuauhtémoc Valdés Olmedo, «Sentido y alcance de la historia de la Universidad en el Centro de Estudios sobre la Universidad», en Memorias del Primer Encuentro de Historia sobre la Universidad (México: CESU-UNAM, 1984), 8-9.

${ }^{15}$ Leticia Pérez Puente y Rosalina Ríos Zúñiga, «Presentación», en De maestros y discípulos. México, siglos XVI-XIX (México: CESU-UNAM, 1998), 9-10.
} 
Como el Seminario mantiene desde hace varias décadas una relación estrecha con el grupo que en la Universidad de Valencia estudia la historia de las universidades españolas y americanas, en cuanto a sus principios teóricos y metodológicos más generales, y las orientaciones académicas más recientes en historia de las universidades europeas, sus contribuciones se orientan en un sentido más amplio. ${ }^{16}$ En esta medida, sus investigaciones han abierto una riquísima veta para analizar las características e implicaciones - económicas, políticas, sociales, culturales- de los estudios superiores en México durante la época colonial y las primeras décadas del siglo XIX. Es de mencionar aquí, entre sus principales aportes, la formación de investigadores dedicados al estudio de la Universidad en la época colonial y la colección de estudios y textos sobre la Real Universidad de México, amén de varios libros colectivos en coedición con editoriales comerciales y universitarias.

Finalmente, en la década de los ochenta, la historia de la educación se convirtió en un campo de trabajo para un grupo heterogéneo de profesionales (filósofos, pedagogos, químicos, antropólogos, entre otros). ${ }^{17}$ Hoy, este campo, se cultiva a lo largo y ancho del país. Sus investigadores conforman un contingente híbrido con distintas trayectorias personales, posiciones desiguales en el establishment académico y, desde luego, diferentes perspectivas teóricas y metodológicas. ${ }^{18}$ De esta guisa, frente a una visión uniforme del desarrollo educativo, surgió una historia de la educación mucho más rica y más compleja.

Sin que hayan desaparecido las formas historiográficas tradicionales, en tres décadas de trabajo se pasó de una forma de hacer historia predominantemente centrada en los acontecimientos a otra que vislumbró estructuras y, de esta, a una cuyo interés mayor es el estudio, plural y diverso, de los procesos pedagógicos y educativos. El trabajo historiográfico, en consecuencia, se desplazó de las estructuras a los actores, del sistema a las escuelas, de los procesos educativos generales a los procesos

\footnotetext{
${ }^{16}$ Mariano Peset Macebo, «Prólogo», en Claustros y estudiantes. Congreso Internacional de Historia de las Universidades Americanas y Españolas en la Edad Moderna (Valencia: Facultad de Derecho-Universidad de Valencia, 1989), XIII-XIV y XIX-XXXI.

${ }^{17}$ Luz Elena Galván Lafarga y Susana Quintanilla Osorio, Historiografía de la Educación (México: Consejo Mexicano de Investigación Educativa (1993), 11 y 38.

${ }^{18}$ Galván y Quintanilla, Historiografía de la Educación, 38. Luz Elena Galván Lafarga, «Un recorrido por la historia de la educación hoy en día», Educar. Revista de Educación, 3 (1997): 8-20.
} 
de la vida escolar, de las ideas a la construcción de discursos, de los casos a las políticas culturales y, de la identidad nacional a la construcción de identidades individuales y colectivas.

La historia de la educación se cultiva hoy en muchas instituciones del país, y lo que pudiera parecer su fortaleza — su profesionalización y la convergencia de distintas disciplinas relacionadas o no con los procesos educativos- es también su debilidad, pues comparte con la historiografía mexicana la mayor crisis intelectual, que para Enrique Florescano radica en

su incapacidad para ofrecer a la nación una historia de la nación. Dividida como está en tantas parcelas como hay historiadores o corrientes historiográficas, se antoja imposible que una de ellas pueda reunir esa miríada de especialidades en un todo coherente, significativo y accesible al lector común. La desvinculación de las instituciones y de los profesores que las integran se observa en el hecho de que ambos son indiferentes a las demandas sociales. No hay nuevas historias dedicadas al público amplio, ni síntesis, ni obras de divulgación que vuelquen al conjunto social el saber acumulado en los centros de investigación. ${ }^{19}$

\section{CONDICIONES DE PRODUCCIÓN DEL CONOCIMIENTO}

El rumbo que tomó la producción de conocimiento en estas penúltima y última décadas quedó marcado por los procesos de institucionalización y de especialización del campo. Los años noventa y los primeros años del siglo XXI nos dan indicios del tránsito más evidente del historiador al especialista legitimado y certificado por variantes del asociacionismo académico, sea que se trate de colegios, academias y distinto tipo de agrupaciones, que serán quienes establezcan las reglas del juego en la escritura de la historia: temáticas, metodologías, enfoques, formatos, aparato crítico. Paulatinamente serán normados y cada vez obedecerán a mayores controles desde el interior de los grupos académicos (baste revisar algunos de los formatos, cada vez más perfeccionados y meticulosos, que se utilizan para dictaminar). La figura de los pares académi-

\footnotetext{
${ }^{19}$ Enrique Florescano, Historia de las historias de la nación mexicana (México: Taurus, 2002), 448-449.
} 
cos, de personas expertas y asociaciones que actúan como árbitros en las premiaciones y concursos, ${ }^{20}$ de los dobles o triples dictámenes ciegos, se impondrán para la sanción de ponencias en los congresos, la publicación de artículos en revistas arbitradas e indexadas, capítulos y libros con el consabido ISBN, que ya no garantiza la calidad del trabajo en cuestión.

A la vuelta de una década, si bien se ha logrado mayor rigurosidad y meticulosidad en la presentación de escritos de diverso tipo con un alto nivel de especialización y de exigencias que actúan como filtros, también es cierto que se ha enclaustrado la producción y el ámbito de incidencia y proyección social. Pareciera que solo se escribe para los pares. Si bien se exploran otras formas de divulgación del conocimiento histórico sobre la educación, a menudo es escaso el contacto con otras realidades externas a la vida académica. Son los órganos evaluadores, de distinta magnitud y nivel de incidencia, los que paulatinamente han ido marcando las reglas del juego sobre las prácticas del historiador de la educación. La exigencia ha redundado en la superespecialización temática con la pérdida de la posibilidad de generar explicaciones globales que acerquen a la comprensión de un determinado proceso.

La necesidad de publicar bajo esas reglas del juego se ha incrementado por las políticas de deshomologación salarial, de manera paralela a los espacios que se han abierto en revistas y congresos, los más, y aun en editoriales comerciales e institucionales, los menos. El problema subsiste en los centros de producción-distribución ampliamente consolidados y en los marginales, entre el historiador ampliamente certificado y «arbitrado" y el que no ha logrado ese estatus; entre la cantidad de publicaciones de dudosa calidad y otras aportaciones.

Ahora bien, en el curso de la década 2002-2012, además de la consolidación de la normativa impuesta, desde lo que pudiéramos llamar neointervencionismo estatal para legitimar la producción en el campo de la historia de la educación, hemos asistido a nuevas condiciones que impactan la producción.

El incremento de posgrados en el campo ha representado el aumento de una mayor cantidad de investigaciones, algunas de las cuales resultan

${ }^{20}$ Wietse de Vries, "Mandarinas en salmuera», Revista de la Educación Superior, XXXVI (142), (2007): 123-130. 
originales y rigurosas, en la medida en que han pasado por distintos filtros a pesar de ser «flor de un día», o bajo el problema de que los nuevos doctores no necesariamente habrán de dedicarse a la investigación si no tienen las condiciones institucionales para ello, aunque también hay que tener presente la proliferación de posgrados que no reúnen las condiciones mínimas de calidad y de producción.

Aquí entran en juego las políticas modernizadoras del gobierno federal que impactaron en la proliferación de los posgrados, donde el propósito central no es necesariamente la formación de recursos para la investigación, sino la necesidad, por un lado, que tienen las propias instituciones de incluir un elevado número de doctores entre su planta académica para lograr su acreditación. Por otro lado, a las personas, la publicación les es útil como recurso de promoción escalafonaria, como recurso de permanencia institucional o de recontratación temporal en un ambiente académico precario. De esta manera se fueron modelando nuevas identidades institucionales, individuales y colectivas, que han marcado, de manera sustancial, el desempeño del oficio del historiador de la educación.

Una de las acciones que han redefinido la orientación y las condiciones de la producción historiográfica son las políticas de evaluación implementadas en el curso de la década, a partir de las cuales se observa una atomización de la vida institucional en relación con el modelo anterior de Universidad: ahora la producción se realiza prioritariamente en los cuerpos académicos.

Una de las políticas establecida por el Consejo Nacional de Ciencia y Tecnología (CONACYT), particularmente relevante en el transcurso de la década, está referida a la internacionalización tendente a la "participación en la emergencia de grupos científicos internacionalizados y conectados con base en capacidades, compartidas o complementarias, de producir conocimientos disciplinarios en lugar de estructurarse en contigüidades espaciales o institucionales». ${ }^{21}$ Esta política, aplicada a los posgrados, a partir de 2005 ha propiciado la adquisición de posgrados en el extranjero en instituciones de prestigio. Certificaciones que integran un capital

\footnotetext{
${ }^{21}$ Sylvie Didou Aupetit y Gerard Etienne, «El Sistema Nacional de Investigadores en 2009. ¿Un vector para la internacionalización de las élites científicas?», Perfiles educativos, XXXIII (132), (2011): 29-47 (31).
} 
académico que, por un lado, fortalece la trayectoria académica acorde con los parámetros del Sistema Nacional de Investigadores dependiente del CONACYT. Por otro lado, en pocos años erige un mundo de diferencias en relación con las generaciones precedentes formadas en la lógica de los posgrados nacionales. Así, estaríamos frente a un fenómeno de cosmopolitización en el contexto globalizado en términos de Beck, en la medida en que los parámetros son supranacionales, generando compromisos y redes de relaciones y de producción de conocimiento distantes y ajenas a las exigencias propias de una sociedad en el contexto de los Estados nacionales. ${ }^{22}$

El aprendizaje de la profesión en estas condiciones sigue los lineamientos del PROMEP (Programa de Mejoramiento del Profesorado), hoy PRODEP (Programa para el Desarrollo Profesional Docente), que plantea la exigencia de que los estudiantes, ya desde la licenciatura, participen en un número determinado de congresos, así como la realización de estancias académicas nacionales e internacionales, como una de las formas de compenetrarse con la práctica del oficio.

\section{ALGUNAS TENDENCIAS Y TEMÁTICAS EMERGENTES}

En el vasto horizonte de la historiografía de la educación en la actualidad, nos interesa destacar lo que se puede inferir como algunas de las tendencias globales emergentes en el campo. ${ }^{23}$ Sin duda hay distintas perspectivas y enfoques. Nuestra visión apunta a la apertura que ha propiciado el posestructuralismo, con su explosión de discursos y pluralidad temática. Valga explicitar que partimos de la concepción de la educación como una práctica histórico-cultural en la idea de lo que Norbert Elias denominó coacciones civilizatorias, donde entra en juego un componente ético y moral que la define. Asimismo, de acuerdo con Santoni

\footnotetext{
${ }^{22}$ Ulrich Beck, La società cosmopolita (Milano: Il Mulino, 2006), 99.

${ }^{23}$ Un análisis puntual producto por producto, derivado de los registros sobre la producción académica, desborda el propósito de este artículo, pero se puede consultar en el libro colectivo María Esther Aguirre Lora (coord.), Historia e historiografía de la educación en México. Hacia un balance 2002-2011 (México: ANUIES-COMIE, 2016, 2 vols.). Este mismo incluye un disco compacto que concentra la bibliohemerografía especializada producida durante la década. Ahí, el lector interesado puede consultar los capítulos referidos al análisis específico de temáticas, procedimientos metodológicos y perspectivas vigentes en la producción académica del campo de la historia e historiografía de la educación en México, en cuanto a archivos, libros y capítulos, artículos y revistas, ponencias presentadas en congresos y tesis de posgrado.
} 
Rugiu consideramos que lo educativo está presente en todos los ámbitos y esferas de la vida social y no se circunscribe a la institución escolar.

\section{Apertura de archivos para la historia de la educación}

En el contexto de la Ley federal de transparencia y acceso a la información pública gubernamental (2002), asistimos a un despliegue en la organización de archivos generales y especializados en educación. Si bien el propósito nunca fue hacer visibles los archivos históricos, la demanda de información y la historia del ciclo vital del documento llevaron a las instituciones a preocuparse no solo por los archivos de trámite, sino también por los documentos inactivos. Ello fue producto de un esfuerzo concurrente en el que participaron tanto el gobierno federal como los gobiernos estatales y municipales, las instituciones educativas y las organizaciones de la sociedad civil interesadas en preservar y difundir nuestro pasado educativo. Lo anterior se tradujo en el fortalecimiento y la apertura de nuevos archivos históricos, tanto en las universidades públicas como otros bajo el cobijo de la Secretaría de Educación Pública. Tal es el caso de las Escuelas Normales. El despliegue ha sido tan amplio y diversificado que ha enriquecido sustancialmente los recursos para la investigación histórica y educativa.

\section{Historias entramadas}

Una de las tendencias emergentes que abre el espectro de perspectivas de indagación es la que se refiere a los estudios comparados en educación. A partir de mediados de los ochenta, los estudios comparados en educación entraron en un importante proceso de renovación, donde Jürgen Schriewer, cuyas aportaciones se introdujeron al mundo de lengua española a través de Miguel A. Pereyra (Universidad de Granada), tiene mucho qué decir al respecto: ha organizado seminarios, programas internacionales de investigación, publicaciones individuales y colectivas, proyectos en los que algunos de nuestros historiadores de la educación y otros estudiosos del campo de la pedagogía comparada han participado. De ahí el interés manifestado por esta línea emergente. 
De esta corriente renovadora han pasado por lo menos dos décadas, y aún falta un largo trecho por recorrer para su consolidación y franca difusión entre las comunidades de historiadores de la educación.

El actual reposicionamiento de los estudios comparados en educación se da en el contexto de la globalización, que necesariamente apunta al asunto de las modernidades, a la búsqueda de nuevos parámetros que aporten explicaciones históricas que vayan más allá del contexto de lo nacional y de lo occidental propiamente dicho, marcos en los que prevalentemente se han desplazado nuestras historias nacionales, las mismas que habían dominado el espacio de las indagaciones en historia de la educación.

La globalización, si bien constituye un fenómeno político-económico, tiene importantes implicaciones en el terreno educativo y cultural, en la medida en que pone de relieve que «hasta los aspectos más aparentemente inconexos de la economía global contemporánea y de los movimientos financieros dependen de conjuntos de estructuras, instituciones y procesos, determinados y controlados políticamente». ${ }^{24}$ La situación ha traído consigo una revisión profunda de conceptos y referentes clave desde los cuales hemos construido las historias de la educación, pues en estos contextos globalizadores asistimos a nuevos deslizamientos que transitan del Estado-Nación individual, que paulatinamente se ha ido adelgazando y perdiendo visibilidad, al territorio de lo transnacional. ${ }^{25}$ Paralelamente se han fortalecido los continuos procesos de homogeneización educativa y cultural que no nos son desconocidos: se pusieron en marcha desde siglos anteriores. Es el movimiento de los nuevos institucionalistas el que marca la condición de los sistemas educativos en sus distintas dimensiones que no son susceptibles de explicarse solamente a través de lo nacional, sino integrando también una perspectiva mundial o global, tocante a

\footnotetext{
${ }^{24}$ Roger Dale, «Globalización: ¿un nuevo mundo para la educación comparada?», en Formación del discurso en la educación comparada, Jürgen Schriewer (comp.) (Barcelona: Pomares, 2002), 69-90 (75).

${ }^{25}$ Dale, «Globalización: ¿un nuevo mundo para la educación comparada?», en Formación del discurso en la educación comparada, 69; N. S. Barbieri, La storia dell'educazione in prospettiva comparata. Riflessioni teoriche e studio di casi: due tradizioni di ricerca a confronto (Padova: CLEUP, 2013).
} 
la modernización en curso, que cada vez compartimos en mayor o menor grado y que aporta referentes para los estudios comparados. ${ }^{26}$

Los estudios comparados primero se aplicaron en el terreno de las ciencias sociales en general y llegaron con cierto retraso al campo de la historia, y más aún de la educación. Puede decirse que, si bien se sigue esgrimiendo su conveniencia y sus dificultades metodológicas, aún hoy hay poco camino andado al respecto. Es posible que estemos más próximos a lo que Kocka llama "historias entramadas», ${ }^{27}$ en la medida en que existen volúmenes colectivos organizados con participaciones nacionales e internacionales sobre un campo en particular, motivados por el propósito de indagar relaciones entre varios procesos que se tocan en algún punto, lo cual es un buen indicio de que estamos en el camino de la comparación. Las publicaciones en torno al Centenario y Bicentenario (de la Independencia y la Revolución Mexicanas, así como de las Independencias latinoamericanas) también ofrecieron un espacio interesante para los Estudios Comparados, como lo muestra el caso de los movimientos independentistas a lo largo de América Latina.

Indudablemente hay un reto, de base, que debemos enfrentar si queremos incursionar en la construcción de una historia global que trascienda las fronteras de lo nacional:

Los historiadores han sido entrenados para basar sus estudios en archivos y fuentes primarias. Por contraste, muchos sociólogos, economistas e incluso cientistas políticos ven su ciencia en términos macrohistóricos y nomotéticos, cuyo fin es obtener generalizaciones. ${ }^{28}$

La misma complejización de las sociedades requiere un arsenal conceptual y metodológico que le permita dar cuenta de ello. Por lo demás, la historia de la educación, desde la perspectiva de los estudios comparados, requiere el soporte de equipos transnacionales e interdisciplinares,

\footnotetext{
${ }^{26}$ John W. Meyer y Francisco O. Ramírez, La institucionalización mundial de la educación», en Formación del discurso en la educación comparada, 92.

${ }^{27}$ Jürgen Kocka, Historia social y conciencia histórica (Madrid: Marcial Pons, 2002) y «Comparison and Beyond", History and Theory, 42 (1), (2003): 39-44 (42).

${ }^{28}$ Georg G. Iggers, Historiography in the Twentieth Century: From Scientific Objectivity to the Postmodern Challenge (Middleton: Wesleyan University Press, 2005), 255.
} 
procesos en los que vamos avanzando, como lo muestran las políticas del CONACYT referidas a la internacionalización, las redes de investigación y los grupos académicos que se han configurado en esta década.

\section{De lo macro a lo micro, y luego a lo macro}

Ya desde el estado del conocimiento de 1993 se percibía el deslizamiento de enfoques que iban de lo macrosocial a lo microsocial, ${ }^{29}$ tendencia que ha continuado incrementándose y, más aún, ha colocado el asunto de la construcción del sujeto en el centro de nuestras preocupaciones. El impacto de las ciencias sociales en los modos de hacer historia de la educación se fue desplazando por la antropología, y específicamente por la etnografía, con lo cual se busca subsanar el olvido del sujeto, perdido entre las macrohistorias, los grandes procesos, las grandes estructuras. El reto metodológico que ello ha planteado es cómo recuperar a las personas en la historia. Esto ha implicado ver hacia los márgenes, hacia los grupos pequeños, hacia la gente común, hacia lo popular, hacia lo local, a lo cual Annales se ha referido como «democratización» de la historia, en la medida que desde hace algunas décadas se ha ido desplazando el interés por la historia de los grandes hombres y grandes eventos a segmentos más circunscritos y anónimos de la población. Este cambio ha implicado cambios de escala en los tiempos, en los espacios, en los procesos.

Por lo anterior, no es casual que los historiadores de la educación en México, en el trayecto de la reciente década, hayan tratado de fortalecer el uso de la historia oral, la búsqueda de las historias de vida, el regreso a las biografías. En ellos el empleo de la microhistoria se ha vuelto recurrente. En él se pueden distinguir distintas perspectivas: una más próxima a la microhistoria italiana, impulsada por Carlo Ginzburg y Giovanni Levi, donde el enfoque sobre un microuniverso revela la complejidad del mundo; otra, más próxima a Luis González y González, que emparenta la microhistoria con el ámbito de los estudios locales, desde una perspectiva romántica.

Algunas de las limitaciones que se pueden apreciar en indagaciones que se rotulan como microhistoria radican en que al reducirse la escala se quedan en ella; otras han dado juego a la escala micro-macro-micro,

\footnotetext{
${ }^{29}$ Weiss, El campo de la investigación educativa en México, 15.
} 
aproximándose a lo pequeño sin olvidar la comprensión de los contextos más amplios que ello posibilita en ambas direcciones, lo micro y lo macro como procesos complementarios, dialécticos, en las explicaciones históricas.

La microhistoria también ha propiciado el tránsito de la esfera pública a la esfera privada, haciendo de la vida cotidiana un campo de observación, próxima a la nouvelle histoire y a filósofos como Agnes Heller, sociólogos, discípulos de Durkheim y de Goffman, e historiadores como Carcopino, que hace varios años plantearon la importancia de recuperar la vida cotidiana. Una tendencia importante en este sentido es la que echa mano de los recursos de la antropología cultural (Geertz), de la semiótica, de la teoría literaria, practicada por antropólogos, sociólogos y literatos.

Por otra parte, nuestra sociedad actual, sometida a los vaivenes del neoliberalismo y de los proyectos globalizadores, estandarizadores de las distintas formas de la vida social y cultural, por fuerza se afianza a la comprensión de la construcción de las identidades colectivas, en un retorno a lo local donde el sujeto deviene en campo de estudio, siendo motivo de lecturas renovadas como la exploración de la subjetividad a partir de la visibilidad de los sentimientos. Por su parte, la biografía histórica adquiere nuevos sentidos, en una amplia gama de tendencias y propuestas metodológicas que van desde la biografía de corte individual hasta la biografía colectiva.

\section{Cultura escolar}

En el contexto del giro cultural (que se ha ido imponiendo sobre las explicaciones de corte socioeconómico y político), uno de los conceptos ampliamente incorporado por los historiadores de la educación, ha sido el de cultura escolar, propuesto por Dominique Julia desde 1993 —en la conferencia de clausura de la XV reunión de la International Standing Conference for the History of Education, en su novedoso texto «La culture scolaire comme objet historique»- que se ha difundido en nuestro país por diversas vías, ${ }^{30}$ destacando las referencias constantes de auto-

\footnotetext{
${ }^{30}$ Margarita Menegus Bornemann y Enrique González González (coords.), Historia de las universidades modernas en Hispanoamérica: métodos y fuentes (México: CESU-UNAM, 1995).
} 
res como Antonio Viñao y Agustín Escolano. En el curso de estos años, esta perspectiva ha ido derivando en líneas de investigación en las que se cruzan la historia de las prácticas escolares, la normativa escolar, la vida cotidiana en la escuela, los estilos de vida de los distintos actores, los artefactos escolares, las culturas de los distintos actores, los manuales escolares, y todo lo que se expresa como vida interna de la escuela que surge in situ, sin perder de vista su relación con el campo más amplio de prácticas culturales en general.

Julia, en esa reunión, hacía un llamado a los historiadores de la educación sobre uno de sus olvidos fundamentales: la indagación de las prácticas escolares, en la medida en que los enfoques que habían prevalecido procedían de las ciencias sociales, y propiciaba un emplazamiento crítico de las pedagogías normativas, que desconocen la vida escolar leída en clave etnográfica y antropológica, como campo de resistencias y tensiones. ${ }^{31}$

Puede decirse que en las últimas dos décadas la investigación de la cultura escolar ha fructificado de tal modo que, con el avance de las nuevas tecnologías y la necesidad de recuperar la memoria, han aparecido, como sujetos y objetos de estudio, los museos escolares, la historia material de la escuela, la iconografía de la escuela como fuente.

Por otra parte, si bien existe interés por la indagación sobre la cultura material plasmada en museos, son pocas las investigaciones que existen al respecto. Una de las prácticas más abonadas en las universidades públicas estatales, y en algunas escuelas normales, ha sido promover y montar exposiciones sobre los instrumentos del saber, como pueden ser laboratorios, manuales y libros escolares, reproducción histórica de espacios áulicos y otros más.

\section{A MODO DE CIERRE}

A partir de nuestra perspectiva de lo histórico-educativo, el horizonte que abordamos en este artículo no se circunscribió ni a lo didáctico ni a lo pedagógico, sino que incluyó indagaciones que tienen que ver con la

\footnotetext{
${ }^{31}$ Dominique Julia, «La cultura escolar como objeto histórico», en Historia de las universidades modernas en Hispanoamérica: métodos y fuentes, 131-153.
} 
construcción de subjetividades en el significado más amplio del término. En este sentido, puede decirse que la mayor parte de la producción de los historiadores de la educación se orienta a la historia de la vida escolar, donde hay logros muy importantes. Sin embargo, aún existe un largo camino por andar en la exploración de otros espacios formativos y en la articulación con otros campos disciplinares. Se trataría de salir del circuito de lo estrictamente educativo y entrar en diálogo con otros campos de especialidad para regresar a él con nuevas preguntas, nuevos arsenales y nuevos interlocutores. Afortunadamente, encontramos iniciativas al respecto. Es importante señalar también que, entre las tareas pendientes para un futuro próximo, mediadas por una actitud incluyente y flexible, habría que distinguir entre los productos de difusión y los de investigación original, así como los producidos por investigadores ya consolidados y los que están en proceso de formación. Esto permitiría lograr un mapa más fino del desarrollo de nuestro campo.

El campo de la historia de la educación, si bien expresa una vitalidad, en consonancia con el espíritu posmoderno se encuentra fragmentado, tanto por la propia expansión disciplinar de nuestros tiempos como por las políticas de evaluación en curso. La universidad occidental, que surgió con una capacidad crítica, se ha ido diluyendo por las exigencias que le impone la globalización. En esta medida es necesario repensar el papel de las humanidades y, en particular, el trabajo de los historiadores de la educación.

\section{Nota sobre los autores:}

María Esther AguirRe Lora. Sus indagaciones se han dirigido hacia dos campos: la historia social y cultural de la formación artística y la nueva historia de la educación, en cuanto a sus desplazamientos paradigmáticos, estrategias metodológicas y fuentes. El Museo Pedagógico de Praga le otorgó la medalla Jan Amos Komenskeho. Es miembro de la Academia Mexicana de Ciencias, de la Sociedad Española de Historia de la Educación, del Centro Italiano per la Ricerca Storico Educativa. Fue presidenta de la Sociedad Mexicana de Historia de la Educación; fue distinguida en la UNAM con el reconocimiento "Sor Juana Inés de la Cruz». Obtuvo el Premio Universidad Nacional 2011 en el área de Investigación en Huma- 
nidades. Pertenece al Sistema Nacional de Investigadores. Entre sus publicaciones más recientes, se pueden mencionar: Ma. Esther Aguirre, Guillermo Hernández, Francisco Pérez, Jesús Adolfo Trujillo (coords.), Educar en el arte. Protagonistas, instituciones y prácticas en el curso del tiempo; y dos más coordinadas por ella en curso de edición: Rememorar los derroteros. La impronta de la formación artística en la UNAM (IISUE, UNAM-Bonilla Artigas, 2015) y Narrar historias de la educación. Crisol y alquimia de un oficio (IISUE, UNAM-Bonilla Artigas, 2015).

Jesús Márouez Carrillo pertenece al Sistema Nacional de Investigadores. Historiador y doctor en Educación, es autor de diez libros y de numerosos artículos y capítulos de libro sobre distintos aspectos de la historia política, social y cultural de Puebla. Colaborador de la prensa, la radio y la televisión, en 1993 fue nombrado Cronista de Número del Consejo de la Crónica de la Ciudad de Puebla, y, en 1995, miembro activo del Seminario de Cultura Mexicana. Pertenece al Consejo Mexicano de Investigación Educativa y a la Sociedad Mexicana de Historia de la Educación. Sus principales campos y líneas de investigación se relacionan con la historia política y cultural de la educación en México y en Puebla, y la historia social y cultural de las imágenes. Actualmente es profesor investigador de tiempo completo. 